\title{
3D MODELLING METHOD OF HIGH ABOVE GROUND ROCK ART PAINTING USING MULTISPECTRAL CAMERA
}

\author{
K. Zainuddin ${ }^{1}$, Z. Z. Majid², M. F. M. Ariff' ${ }^{2}$ K. M. Idris ${ }^{2}$, N. Darwin ${ }^{2}$ \\ ${ }^{1}$ Centre of Study for Surveying Science \& Geomatics, Faculty of Architecture, Planning \& Surveying Universiti Teknologi MARA, \\ MALAYSIA -khairul760@uitm.edu.my \\ ${ }^{2}$ Geospatial Imaging and Information Research Group, Universiti Teknologi Malaysia, MALAYSIA - (zulkeplimajid, mfaridma, \\ khairulnizami, norhadija2) @utm.my
}

\section{Commission II}

KEY WORDS: 3D Modelling, Multispectral Image, Close-range Photogrammetry, Rock Art

\begin{abstract}
:
This paper presents a developed methodology of 3D modelling of rock art painting located at high above shelter floor based on close-range photogrammetric technique. Instead of using elevated devices to reach inaccessible paintings due to high elevation, the developed method proposed a simple technique. The camera was mounted on an expandable pole to acquire the panel with normal and tilted camera settings. Due to inaccessibility to place the control point marker on the panel surface, the distribution of photo control was positioned below the panel. Based on the configuration, the image of the panel was acquired using the low-spatial resolution multispectral camera in a two elevated strips position that imitates the aerial photogrammetry flight line. The camera was set parallelly to the painting panel on both strips, with additional tilted geometry included at the upper strip of the camera block. The acquired multispectral images were then processed using commercial SfM photogrammetry software to generate a 3D point cloud. The accuracy of constructed point cloud was then analysed by comparing it with the point cloud generated using a terrestrial laser scanner (TLS). The result has shown that the multispectral 3D point cloud has a small deviation against the TLS point cloud. The mean deviation was $-0.43 \mathrm{~mm}$, indicating a slight downscaled on the multispectral point cloud.
\end{abstract}

\section{INTRODUCTION}

Rock art is a piece of historical heritage painted or carved by prehistoric peoples thousands of years ago. Because the paintings have been around for a long time, they have been subjected to natural factors like weather, animals, and microorganisms and the risk of being vandalised by people. Rock art is thought of as a fragile natural heritage object. It deteriorates over time to the factors above, so documenting the motifs in digital form is vital for future research, reference, and dissemination (Bendicho et al., 2017).

Documenting rock art in a digital three-dimensional (3D) model has become demanding in cultural heritage. The method is also suitable for visualisation, inventory, and measurement purpose. The 3D model can be generated either by the range-based or image-based method. The range-based method utilises an active light detector to measure the $3 \mathrm{D}$ point coordinate of the observed object (Peña-Villasenín et al., 2019). Terrestrial laser scanner (TLS) is one of the examples of the active light detector, producing millions of points known as point cloud in a single snap. The point cloud scanned from different stations are required to be registered to produce a completed $3 \mathrm{D}$ model of a measured object. However, even though TLS can generate a 3D model in a short period, the cost of employing the device in rock art projects is also high (Jalandoni, 2021).

In contrast, the image-based is a passive light method that uses a camera to capture and store the object in a two-dimensional (2D) image. The object points on the $2 \mathrm{D}$ image are projected to the 3D space through the mathematical equation in constructing the 3D model. The estimation of the camera position and object point can be calculated automatically with Structure-fromMotion (SfM) photogrammetry. SfM has become dominant in cultural heritage documentation, including rock art, since it operated without in-depth technicality of photo orientation procedure (Jalandoni, 2021).

SfM generates the 3D model by estimating the photo orientation through a sparse point cloud or tie point construction. During this stage, the quality of the image orientation should be statistically analysed regarding their precision by removing the outliers due to wrong point projection and adjusting the estimated tie point coordinate and image orientation elements by bundle adjustment. After that, the dense point cloud will be generated, meshed, and texturised to create a photorealistic 3D model.

Besides using colour images for photorealistic 3D documentation, multispectral images are also utilised to generate a 3D model in cultural heritage applications (Adamopoulos et al., 2020; Mathys et al., 2019; TorresMartínez et al., 2017; Zainuddin et al., 2019). The remote sensing-based sensor extends the analysis of cultural heritage objects in terms of their radiometric and spectral analysis (Liang, 2012; Torres-Martínez et al., 2017). Therefore, the analysis can be performed on the invisible spectrum of the recorded painting.

Planning for data acquisition is essential in the image-based 3D modelling pipeline. Image network geometry should be carefully designed prior to data acquisition for proper camera placement according to the $3 \times 3$ rule of photogrammetric documentation (Waldhäusl \& Ogleby, 1994). According to the geometric rule section, the camera should be positioned to cover a complete object with a suitable image overlap greater than $60 \%$ and incorporate standard stereoscopic geometry. The object point will be visible in at least two to three photographs within the mentioned camera configuration. 
Ground control points (GCP) are also required in SfM processing for model scaling and georeference. The GCPs should be well distributed and frequently located around the object to minimise model distortion. This method is appropriate when the rock art is in an easily accessible area. However, not all rock arts are reachable by human height without elevated apparatus. Additionally, some shelters are located at remote areas with limited access, making mobilising such tools complicated and incurring the project cost.

For instance, the Gua Tambun rock art shelter in Ipoh, Perak, Malaysia, is located approximately $50 \mathrm{~m}$ above ground level (Figure 1). The shelter base is only accessible via a walk through a small jungle and climbs up a narrow and steep manmade concrete staircase. Meanwhile, the paintings are positioned on vertical wall panels ranging from $6 \mathrm{~m}$ to $18 \mathrm{~m}$ above the shelter floor. As a result, mobilising additional devices to reach the high elevated shelter can be challenging.

This paper presents the 3D modelling method involving highabove-ground paintings acquired based on normal human height using a low spatial resolution multispectral camera. In developing the method, several constraints were considered, including the capability and limitation of the multispectral sensor and processing software and the physical size of the painting panel.

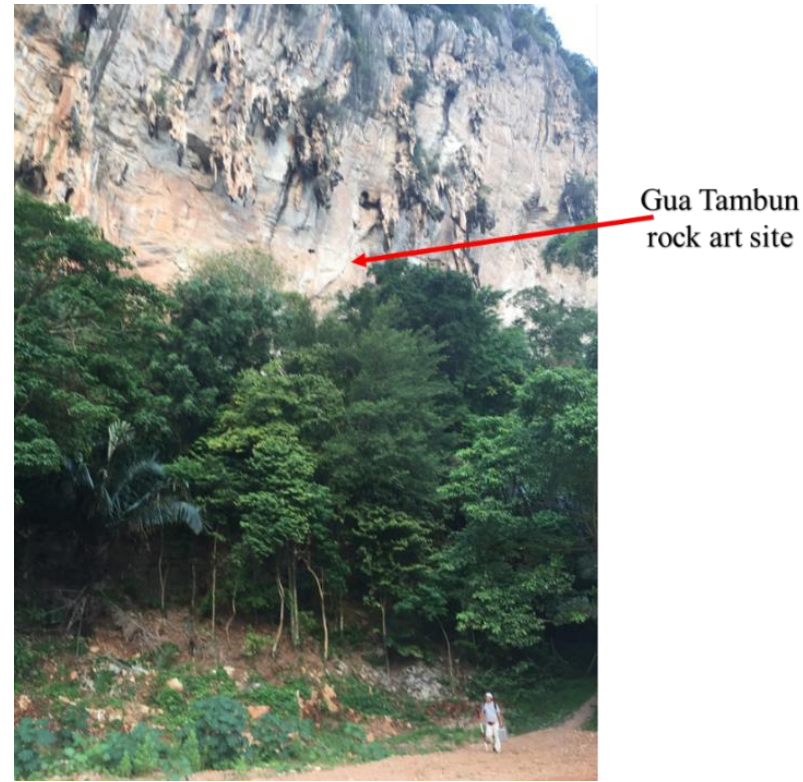

Figure 1. Gua Tambun rock art site.

\section{DEVELOPED METHODOLOGY}

The developed methodology aims to analyse the geometric accuracy of a $3 \mathrm{D}$ point cloud where the images were acquired from limited human height and control points distribution (Figure 3). The experiment was conducted at the main panel of Gua Tambun rock art, where the dimensions are approximately $18 \mathrm{~m}$ wide by $12 \mathrm{~m}$ high and about $6 \mathrm{~m}$ above the cave floor. Due to inaccessibility to place the control point for such elevation, the developed method experiments limited GCP distribution, which was placed at a low level of the panel approximately $3 \mathrm{~m}$ above the shelter floor.

The developed method also acquired images using low spatial resolution multispectral camera. The camera is a miniature multispectral camera that captures five discrete bands in a single snap consisting of three visible (red, green, and blue - RGB) and two invisible wavelengths (red-edge and near infra-red). The camera's sensor size is $4.8 \times 3.6 \mathrm{~mm}$, with $3.75 \mu \mathrm{m}$ of pixel size producing $1280 \times 960$ pixels resolution image. The manufacturer specifies that the sensor's ground sample distance (GSD) is approximately $8 \mathrm{~cm}$ at a $120 \mathrm{~m}$ camera-to-object distance.

\subsection{Data Acquisition}

Proper planning was implemented to ensure the multispectral camera captured the painting's panel according to the $3 \times 3$ rule for geometric documentation. The camera network was designed with two different elevations of camera strips to satisfy $80 \%$ image overlap between images on the same strip and $60 \%$ between strips. The lower strip was set to acquire the panel at an elevation of $1.5 \mathrm{~m}$ by mounting the multispectral sensor on a normal camera tripod. Meanwhile, the upper strip was set at $4.1 \mathrm{~m}$ height to capture the upper panel area. Due to the height limitation of the normal camera tripod, an expandable pole was utilised to mount the multispectral camera to reach the upper part of the panel (Figure 2).

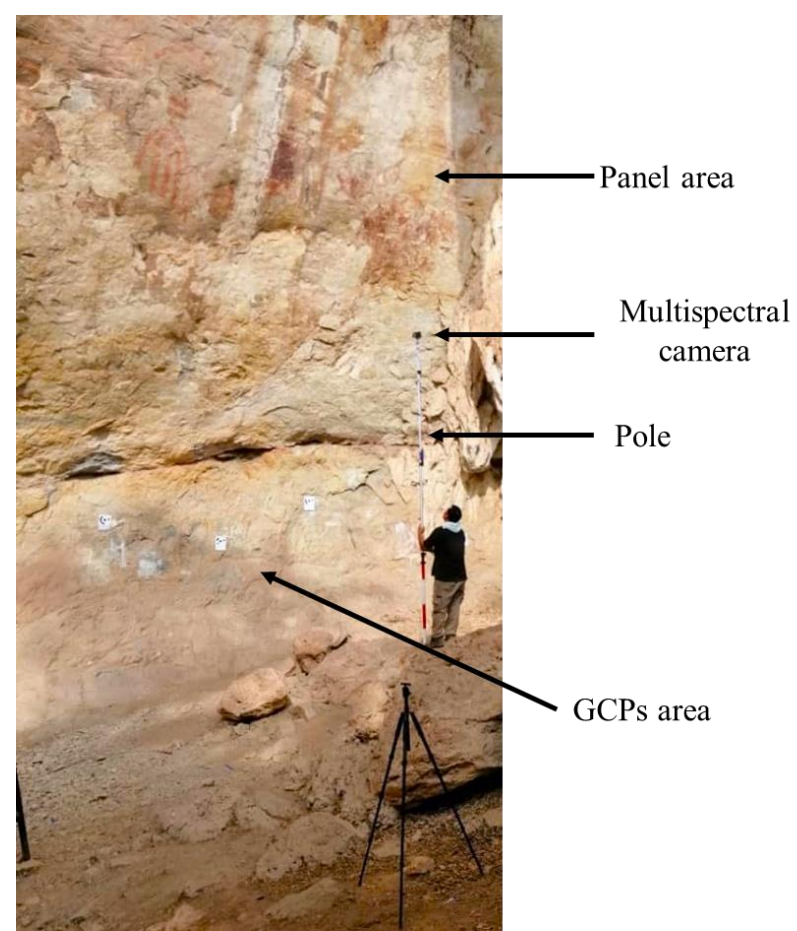

Figure 2. Camera setup with an expandable pole.

The normal camera geometry represented by the red rectangular box in Figure 3 was set at both elevated strips to acquire the panel. The camera was placed parallel to the panel wall during data capture. The configuration of using normal camera geometry ensured the successful image matching through the SfM orientation procedure since the method required a good and large image overlap.

However, the configured setting did not cover the upper part at the $18 \mathrm{~m}$ elevation of the painting panel. In this study, the tilted camera geometry (yellow rectangle box) was set to capture the upper part of the panel by locating the camera at the elevation of the upper strip. By including the tilted camera on the camera block, the area coverage can be extended to cover the $18 \mathrm{~m}$ height of the rock art panel. A few convergent geometry cameras (blue and orange rectangle boxes) were also included 


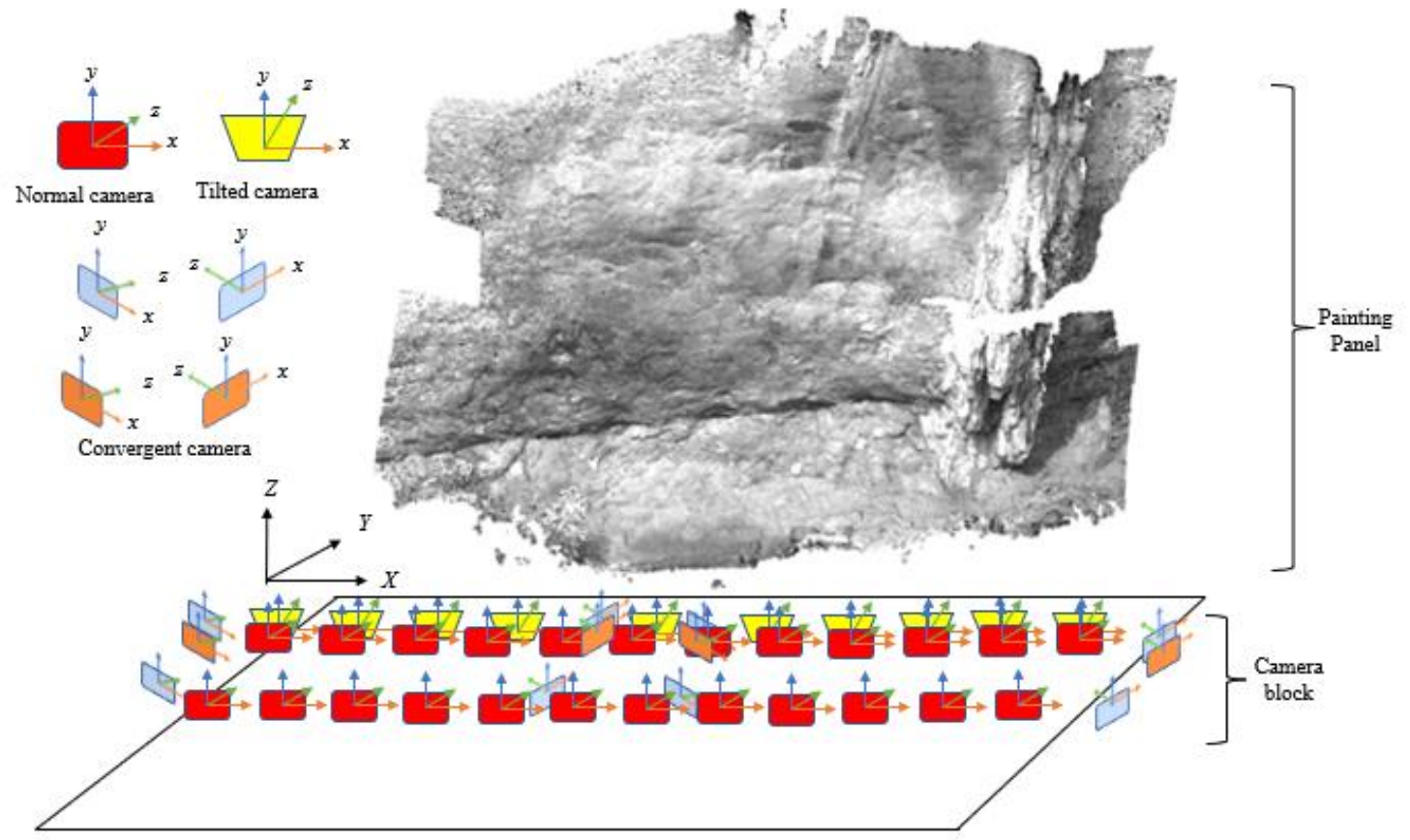

Figure 3. Configuration for data acquisition.

on each camera strip, including on the tilted geometry strip to strengthen the intersection of light rays during orientation and avoid the block deformation of constructed point cloud (Nocerino et al., 2014).

The camera base between stations was set at $2 \mathrm{~m}$ apart and marked on the shelter floor to assist the camera placement during photography sessions. Additionally, the camera was set to capture the painting panel at a $10 \mathrm{~m}$ distance, considering the structure of the cave panel to obtain a good GSD. Based on the developed configuration, the estimated GSD was about $6.8 \mathrm{~mm} / \mathrm{pixel}$, with an expected spatial accuracy of approximately $13 \mathrm{~mm}$ based on the assumed standard error of half-pixel (Barazzetti et al., 2010).

\subsection{Photogrammetric Control Point}

The photogrammetric control was used to scale and orientate the constructed 3D model during processing. However, the high vertical panel structure limits the accessibility to attach the ground control marker for photogrammetric control. Thus, the developed method distributed the photogrammetric control at a lower elevation of rock panel, where there are no paintings available, as shown in Figure 4 indicated by the blue flag.

The coordinate of control points was measured using a reflectorless total station based on three-stations intersection method involving 23 markers. The measured control point coordinate was later adjusted by least-square adjustment, and the reported accuracy was $0.33 \mathrm{~mm}$, indicating a high photo control precision for photogrammetric processing. However, only 11 measured points were used for control and accuracy assessment, while the rest of the 12 points were located outside the panel area.

\subsection{D modelling}

A total of 270 multispectral images were acquired based on the configured method consisting of 54 camera stations located at two different elevations of camera strips. The dataset was then processed using Agisoft Metashape software to generate the 3D model. The default band setting was selected as the primary channel in Metashape to allow the software to orientate all the discrete bands.

The coordinate of the control points was then imported, and their respective location on corresponding images was located to perform bundle adjustment. All the noise was removed statistically using the gradual selection tool based on the step suggested by Mayer \& Kersten (2018). After removing the noise on each step by gradual selection tool, the bundle adjustment procedure was performed to improve tie point coordinate and image orientation. The $3 \mathrm{D}$ modelling procedure was followed by dense point cloud construction and mesh modelling. This paper analysed the constructed dense point cloud by exported to CloudCompare for accuracy assessment.

The same rock art panel was also acquired and modelled using Topcon GLS-2000 terrestrial laser scanner (TLS) for accuracy assessment reference. The point spacing was set at $6.3 \mathrm{~mm}$ based on a $20 \mathrm{~m}$ distance of observation. The acquired data from three scan stations were registered according to the standard protocol of the TLS point cloud procedure. The 3D point cloud was then exported to CloudCompare, which was used as a reference dataset to assess the geometric accuracy of the multispectral 3D model. Both multispectral and TLS 3D models were mutually aligned into a common coordinate system using control points coordinate before exported to CloudCompare. 


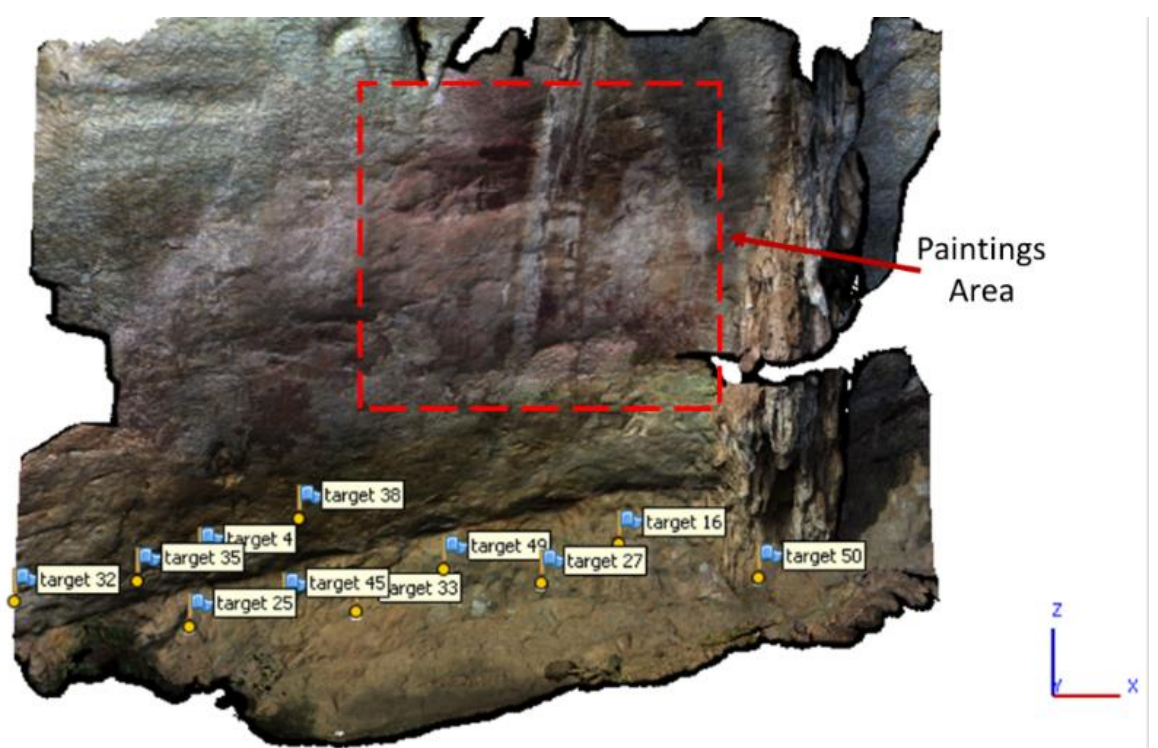

Figure 4. Location of painting panel and control points.

\section{RESULT AND DISCUSSION}

The analysis of the developed procedure initially evaluates the precision of the image orientation of multispectral images. The residual error reported by Metashape shows that the average RMS was 0.52 pixels while the standard deviation was 0.3 pixels. The result indicates that the multispectral image can be aligned precisely even with low spatial features on their images. Within precise image orientation, then each image point can be projected accurately to the $3 \mathrm{D}$ space.

The main analysis assessed the geometric accuracy of the dense point cloud constructed by the multispectral image compared with the TLS dataset. The assessment focused on quantifying the discrepancies between datasets since the images were acquired with tilted geometry and limited control point distribution. Since multispectral consisted of five different bands, all the point clouds of respective bands were evaluated. Due to the identical result of point cloud accuracy of all bands, this paper only reports the outcome of the blue band.

The accuracy evaluation quantifies the distance error produced by the multispectral point cloud. Figure 5 illustrates the discrepancies between multispectral and TLS point clouds analysed using Multiscale Model-to-Model (M3C2) in CloudCompare. The GSD obtained from photogrammetric processing was $6.33 \mathrm{~mm} /$ pixel. The colour scale was set to reflect the GSD obtained from orientation in Metashape, with blue representing one GSD $(6.33 \mathrm{~mm} /$ pixel), yellow representing two GSD $(12.66 \mathrm{~mm} /$ pixel), green representing three GSD $(18.99 \mathrm{~mm} / \mathrm{pixel})$, and red representing all points exceeding three times GSD.

The Gaussian histogram shows the numerical analysis for point cloud deviation, where the mean deviation of $-0.43 \mathrm{~mm}$, as illustrated in Figure 6. The mean deviation indicates that the multispectral 3D point cloud was slightly downscaled. The standard deviation of distance was $9.24 \mathrm{~mm}$, which was considered acceptable because it was less than the two standard deviation threshold. Additionally, symmetry and a sharp histogram support the result, indicating a higher frequency of small point deviations in both datasets. Missing data most likely caused the red colouration on the 3D point cloud due to their location at the model's edge.

\section{CONCLUSION}

The study had successfully accessed the geometric accuracy of the generated 3D point cloud using the developed method. The graphical and numerical analysis have shown that the point cloud generated is comparable to that derived from TLS, even using the low-spatial resolution multispectral images. The photo orientation report from the software also indicates that features on low-spatial multispectral can be matched with decent precision. The image point can be precisely projected to $3 \mathrm{D}$ space with a good orientation to constructing an accurate $3 \mathrm{D}$ dense point cloud.

The inclusion of the tilted image in two elevated normal camera strips has successfully extended the image coverage up to $18 \mathrm{~m}$ of the painting panel. The slight deviation of dense point cloud compared to TLS derived point cloud has shown that the tilted configuration and limited control point distribution do not deform the 3D point cloud, especially at the upper part of the panel, where no control point can be distributed on such elevation.

The experiment has shown that the developed method is suitable for acquiring the image of an object situated at a high elevation from a low-level position. Based on this experiment, the same method can be tested on similar situations such as building and transportation structures. Other sensors should be examined using the developed method to verify the suitability of the developed method. 


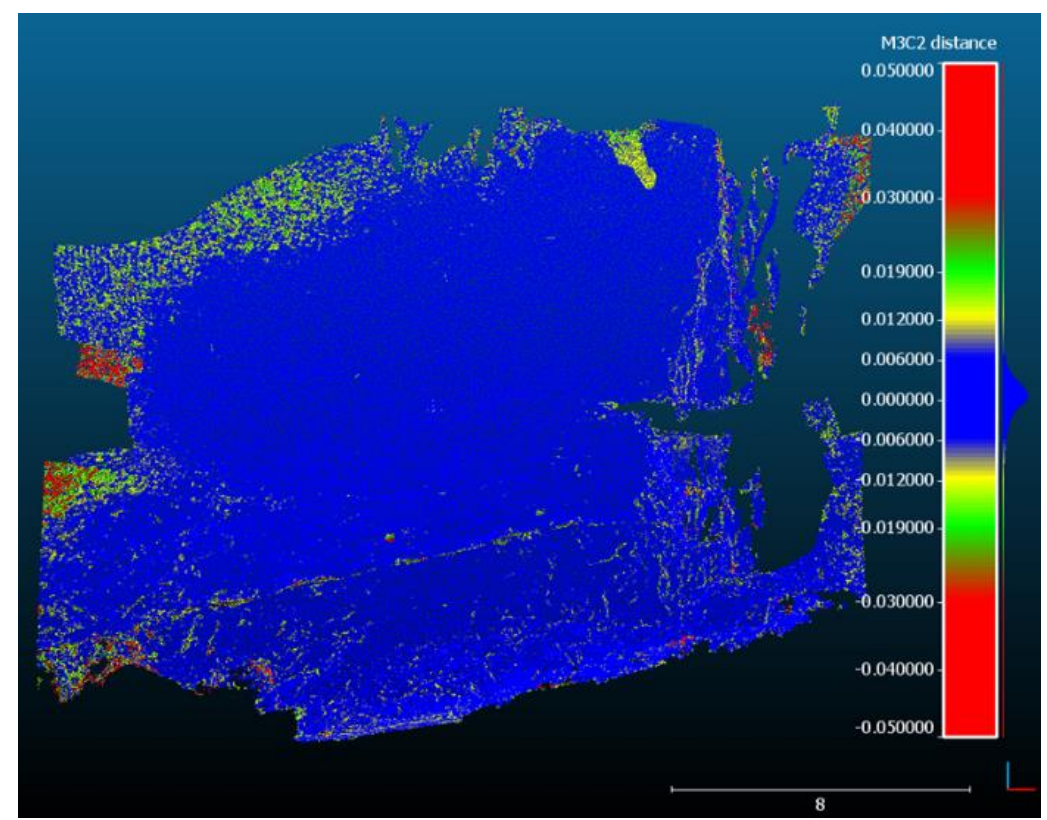

Figure 5. Distance discrepancy between multispectral and TLS point cloud.

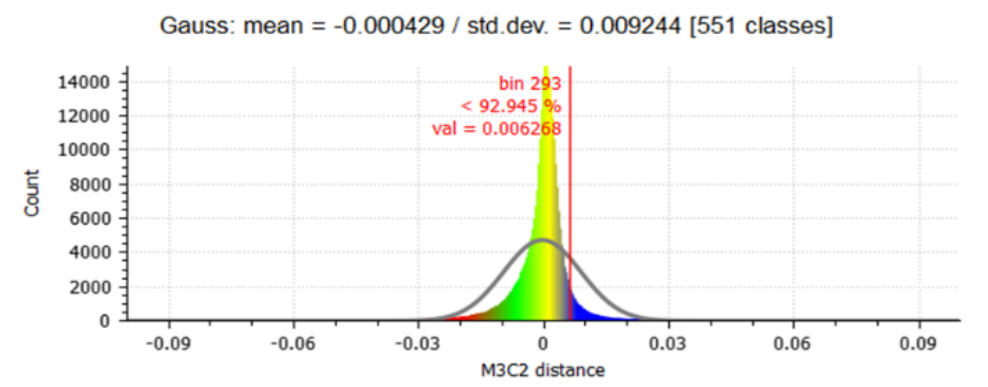

Figure 6. The numerical result of distance discrepancy between multispectral and TLS point cloud.

\section{ACKNOWLEDGEMENTS}

The experiment has been financially supported by Universiti Teknologi Malaysia under the Research University Grant Scheme UTM Fundamental Research Proposal (Q.J120000.2552.21H09, and the Ministry of Higher Education of Malaysia under Fundamental Research Grant Scheme 2021 (R.J130000.7852.5F448). The authors highly appreciate to Department of National Heritage Malaysia for providing the experiment site. Also, the authors highly thanks Mr Anuar bin Aspuri and Mr Mohd Faizi bin Mohd Salleh (UTM-FABU) for data collection.

\section{REFERENCES}

Adamopoulos, E., Bovero, A., \& Rinaudo, F. (2020). Imagebased metric heritage modeling in the near-infrared spectrum. Heritage Science, 8(1), 1-12. https://doi.org/10.1186/s40494020-00397-w

Barazzetti, L., Scaioni, M., \& Remondino, F. (2010). Orientation and 3D modelling from markerless terrestrial images: Combining accuracy with automation. Photogrammetric Record, 25(132), 356-381. https://doi.org/10.1111/j.1477-9730.2010.00599.x
Bendicho, V. M. L.-M., Gutiérrez, M. F., \& Pintado, J. O. (2017). Holistic Approaches to the Comprehensive Management of Rock Art in the Digital Age. In M. L. Vincent, V. M. López-Menchero Bendicho, M. Ioannides, \& T. E. Levy (Eds.), Heritage and Archaeology in the Digital Age: Acquisition, Curation, and Dissemination of Spatial Cultural Heritage Data (pp. 27-47). Springer International Publishing. https://doi.org/10.1007/978-3-319-65370-9_2

Jalandoni, A. (2021). An overview of remote sensing deliverables for rock art research. Quaternary International, 572, 131-138. https://doi.org/10.1016/j.quaint.2019.10.008

Liang, H. (2012). Advances in multispectral and hyperspectral imaging for archaeology and art conservation. Applied Physics A: Materials Science and Processing, 106(2), 309-323. https://doi.org/10.1007/s00339-011-6689-1

Mathys, A., Jadinon, R., \& Hallot, P. (2019). Exploiting 3D multispectral texture for a better feature identification for cultural heritage. ISPRS Annals of the Photogrammetry, Remote Sensing and Spatial Information Sciences, 4(2/W6), 91-97. https://doi.org/10.5194/isprs-annals-IV-2-W6-91-2019

Nocerino, E., Menna, F., \& Remondino, F. (2014). Accuracy of typical photogrammetric networks in cultural heritage 3D 
modeling projects. International Archives of the Photogrammetry, Remote Sensing and Spatial Information Sciences - ISPRS Archives, 40(5), 465-472. https://doi.org/10.5194/isprsarchives-XL-5-465-2014

Peña-Villasenín, S., Gil-Docampo, M., \& Ortiz-Sanz, J. (2019). Professional SfM and TLS vs a simple SfM photogrammetry for 3D modelling of rock art and radiance scaling shading in engraving detection. Journal of Cultural Heritage, 37, 238-246. https://doi.org/10.1016/j.culher.2018.10.009

Torres-Martínez, J. A., Sánchez-Aparicio, L. J., HernándezLópez, D., \& González-Aguilera, D. (2017). Combining geometrical and radiometrical features in the evaluation of rock art paintings. Digital Applications in Archaeology and Cultural Heritage, 5(March), 10-20. https://doi.org/10.1016/j.daach.2017.04.001

Waldhäusl, P., \& Ogleby, C. L. (1994). 3 x 3 rules for simple photogrammetric documentation of architecture. International Archives of Photogrammetry and Remote Sensing, 30, 426-429.

Zainuddin, K., Majid, Z., Ariff, M. F. M., Idris, K. M., Abbas, M. A., \& Darwin, N. (2019). 3D Modeling for Rock Art Documentation using Lightweight Multispectral Camera. The International Archives of the Photogrammetry, Remote Sensing and Spatial Information Sciences, XLII(February), 6-8. 